PROCEEDINGS OF THE

AMERICAN MATHEMATICAL SOCIETY

Volume 139, Number 10, October 2011, Pages 3697-3706

S 0002-9939(2011)10772-X

Article electronically published on February 21, 2011

\title{
A DIFFERENTIAL GEOMETRIC CHARACTERIZATION OF THE CAYLEY HYPERSURFACE
}

\author{
ZEJUN HU, CECE LI, AND DONG ZHANG \\ (Communicated by Jianguo Cao)
}

\begin{abstract}
The so-called Cayley hypersurface, constructed by Eastwood and Ezhov, is a higher-dimensional extension of the classical Cayley surface. In this paper, we establish a differential geometric characterization of the Cayley hypersurface, which is an answer to Eastwood and Ezhov's question.
\end{abstract}

\section{INTRODUCTION}

Let $\mathbb{R}^{n+1}$ be the $(n+1)$-dimensional real unimodular-affine space equipped with its canonical flat connection $D$ and a parallel volume form $\theta$. For a non-degenerate equiaffine hypersurface $M$ of $\mathbb{R}^{n+1}$, the affine normal $\xi$ induces an affine connection $\nabla$ and a semi-Riemannian affine metric $h$. Let $C=\nabla h$ be the cubic form. Then we have the Pick-Berwald theorem which states that $C=0$ implies that $M$ is locally a non-degenerate quadric. Besides non-degenerate quadrics, the next simplest one might be the Cayley surface in $\mathbb{R}^{3}$, given by

$$
x_{3}=x_{1} x_{2}-\frac{1}{3} x_{1}^{3},
$$

which is non-degenerate and affine homogeneous ([14, p. 119). In [13, Nomizu and Pinkall showed that the Cayley surface can be characterized, up to an affine congruence, as the unique affine surface whose cubic form is non-zero and is parallel relative to the induced affine connection, i.e., $C \neq 0$ and $\nabla C=0$.

For general dimensions, Eastwood and Ezhov 7 exhibited a homogeneous hypersurface in $\mathbb{R}^{n+1}$, named as Cayley hypersurface, defined by

$$
\Phi\left(x_{1}, \cdots, x_{n+1}\right) \equiv \sum_{k=1}^{n+1}(-1)^{k} \frac{1}{k} \sum_{i_{1}+\cdots+i_{k}=n+1} x_{i_{1}} x_{i_{2}} \cdots x_{i_{k}}=0 .
$$

This is the Cayley surface when $n=2$. Obviously, the generalized Cayley hypersurface is the graph of $x_{n+1}=\Psi\left(x_{1}, \cdots, x_{n}\right)$, where $\Psi$ is a polynomial function of

Received by the editors April 29, 2010 and, in revised form, August 26, 2010.

2010 Mathematics Subject Classification. Primary 53A15; Secondary 53B25, 53B30.

Key words and phrases. Cayley hypersurface, differential geometric characterization, parallel cubic form.

This project was supported by grants of the NSFC (No. 10671181 and No. 11071225).

(C)2011 American Mathematical Society Reverts to public domain 28 years from publication 3697 
degree $(n+1)$ in $\mathbb{R}^{n}$ defined by

$$
\Psi\left(x_{1}, \cdots, x_{n}\right) \equiv \sum_{k=2}^{n+1}(-1)^{k} \frac{1}{k} \sum_{i_{1}+\cdots+i_{k}=n+1} x_{i_{1}} x_{i_{2}} \cdots x_{i_{k}} .
$$

Eastwood and Ezhov have shown that the Cayley hypersurface has remarkable properties:

Proposition ([7]). For an n-dimensional Cayley hypersurface, the following hold:

(a) It is affine homogeneous and has vanishing Pick invariant.

(b) It is uniquely ruled by $\left\lfloor\frac{n}{2}\right\rfloor$ planes.

(c) Its affine normals are everywhere parallel.

(d) Its affine automorphism group contains a transitive abelian subgroup.

(e) The isotropy subgroup of its affine automorphism group is 1-dimensional.

Eastwood and Ezhov [7] further conjectured that the last three properties of the Proposition should characterize the Cayley hypersurface. This conjecture was proved by Choi and Kim 2 under the additional condition that the domain in $\mathbb{R}^{n+1}$, being bounded by the hypersurface which is expressed as a graph of a function defined on $\mathbb{R}^{n}$, is affine homogeneous. On the other hand, Eastwood and Ezhov [7] raised an interesting question as follows:

How do we extend Nomizu and Pinkall's differential geometric characterization of the Cayley surface to the higher-dimensional Cayley hypersurface?

In this paper, we will show that, as an improper affine hypersphere with vanishing Pick invariant, the Cayley hypersurface has a flat affine metric and satisfies $\hat{\nabla} C=0$ and $K^{n-1} \neq 0$, where $\hat{\nabla}$ is the Levi-Civita connection of the affine metric and $K$ is the difference tensor defined by $K(X, Y)=K_{X} Y=\nabla_{X} Y-\hat{\nabla}_{X} Y$. Most importantly, we will show that the converse holds. This establishes a differential geometric characterization for the Cayley hypersurface and gives an affirmative answer to Eastwood and Ezhov's question. Precisely, we will prove the following.

Main Theorem. Let $M$ be a non-degenerate affine hypersurface of $\mathbb{R}^{n+1}$ with vanishing Pick invariant. If $\hat{\nabla} C=0, K^{n-1} \neq 0$ and the affine metric $h$ is of constant sectional curvature, then $M$ is locally affine equivalent to the Cayley hypersurface.

Remark 1.1. To make a comparison with Nomizu and Pinkall's characterization of the Cayley surface, we point out that it was shown in 4 and 15 , that a hypersurface satisfying $C \neq 0$ and $\nabla C=0$ is a graph immersion of a polynomial function of degree at most 3 . Hence we cannot use the condition $\nabla C=0$ to characterize the higher-dimensional Cayley hypersurface. Related to the Main Theorem, we mention an interesting result of F. Dillen and L. Vrancken 5 which states that the conditions $\nabla K=0$ and $K^{n-1} \neq 0$ indeed characterize a hypersurface of $\mathbb{R}^{n+1}$, which is also a flat improper affine hypersphere and is given by $x_{n+1}=G\left(x_{1}, \cdots, x_{n}\right)$, where

$$
G\left(x_{1}, \cdots, x_{n}\right)=\sum_{k=2}^{n+1} \frac{(-2)^{k-2}}{k !} \sum_{i_{1}+\cdots+i_{k}=n+1} x_{i_{1}} x_{i_{2}} \cdots x_{i_{k}} .
$$

Remark 1.2. The Cayley hypersurface is an improper affine hypersphere which has constant affine sectional curvature and vanishing Pick invariant. Closely related to this fact, we would point out that L. Vrancken [16] has classified affine hyperspheres with constant affine sectional curvature and non-vanishing Pick invariant. 
Remark 1.3. Many researches have been carried out with the condition $\hat{\nabla} C=0$. For locally strongly convex (i.e. $h$ is definite) affine hypersurfaces with $\hat{\nabla} C=0$, after continuous efforts by F. Dillen, L. Vrancken et al. and obtaining the classifications for low dimensions ([12, 3], 6], 9], 10]), Z.J. Hu, H. Li and L. Vrancken [1] eventually obtained a complete classification. For general non-degenerate affine hypersurfaces with $\hat{\nabla} C=0$ and the fact that $h$ is not definite, the classification problem remains open. Besides [12] for $n=2$, the first two authors recently solved the problem for the 3 -dimensional case $[8$.

\section{Differential geometric Properties of CAYley hypersurfaces}

In this section, we will show that the Cayley hypersurface has the following remarkable differential geometric properties:

Proposition 2.1. The Cayley hypersurface in $\mathbb{R}^{n+1}$ is a flat improper affine hypersphere which has the properties that $\hat{\nabla} C=0$ and $K^{n-1} \neq 0$.

First of all, we will briefly recall some basic formulas in affine differential geometry. For more details, we refer to [14. For a non-degenerate affine hypersurface $F: M \hookrightarrow \mathbb{R}^{n+1}$, let $S$ be the affine shape operator (or Weingarten operator). Then, for any tangent vector fields $X$ and $Y$, we have the Gauss and Weingarten formulas

$$
D_{X} Y=\nabla_{X} Y+h(X, Y) \xi, \quad D_{X} \xi=-S X .
$$

$M$ is called an affine hypersphere if $S=\lambda i d$, and one easily proves that $\lambda=$ const for $n \geq 2$. $F$ is called a proper affine hypersphere if $\lambda \neq 0$ and an improper affine hypersphere if $\lambda=0$. For an improper affine hypersphere the affine normal $\xi$ is constant.

The curvature tensor $\hat{R}$ of $\hat{\nabla}$ is related to $S$ and $K$ by the Gauss equation

$\hat{R}(X, Y) Z=\frac{1}{2}[h(Y, Z) S X-h(X, Z) S Y+h(S Y, Z) X-h(S X, Z) Y]-\left[K_{X}, K_{Y}\right] Z$.

In particular, for affine hyperspheres we have $S=\lambda i d$, and thus

$$
\hat{R}(X, Y) Z=\lambda(h(Y, Z) X-h(X, Z) Y)-\left[K_{X}, K_{Y}\right] Z .
$$

Then we have the relation

$$
\chi=\lambda+J
$$

where $J=\frac{1}{n(n-1)} h(K, K)$ is the so-called Pick invariant and $\chi$ is the normalized scalar curvature of the affine metric $h$. From the well-known relation $C(X, Y, Z)=$ $-2 h(K(X, Y), Z)$ we see that $\hat{\nabla} C=0$ is equivalent to $\hat{\nabla} K=0$. Moreover, $K$ satisfies the apolarity condition, i.e., $\operatorname{tr} K_{X}=0$ for all $X$, and it has the property that $h(K(X, Y), Z)$ is totally symmetric in $X, Y$ and $Z$.

To prove Proposition 2.1, we notice that from the affine homogeneity of the Cayley hypersurface, it is sufficient to show that the conclusion holds around some point $p=\left(x_{1}^{0}, x_{2}^{0}, \cdots, x_{n+1}^{0}\right) \in \mathbb{R}^{n+1}$, where $x_{i}^{0}(i=1,2, \cdots, n+1)$ are all sufficient small positive numbers.

We will need the following. 
Lemma 2.1. The Cayley hypersurface (1) can be parameterized, around $p \in \mathbb{R}^{n+1}$, by

$$
\begin{aligned}
& x_{1}=v_{1}, x_{2}=v_{2}+\frac{1}{2 !} v_{1}^{2}, \cdots, x_{n}=\sum_{i_{1}+2 i_{2}+\cdots+n i_{n}=n} \frac{1}{i_{1} ! i_{2} ! \cdots i_{n} !} v_{1}^{i_{1}} v_{2}^{i_{2}} \cdots v_{n}^{i_{n}}, \\
& x_{n+1}=\sum_{i_{1}+2 i_{2}+\cdots+n i_{n}=n+1} \frac{1}{i_{1} ! i_{2} ! \cdots i_{n} !} v_{1}^{i_{1}} v_{2}^{i_{2}} \cdots v_{n}^{i_{n}},
\end{aligned}
$$

where $v_{i}(i=1,2, \cdots, n)$ are all sufficient small positive numbers.

Proof. It is sufficient to show that the $(n+1)$ functions $\left\{x_{i}\right\}_{1}^{n+1}$ in (5) satisfy the relation

$$
x_{n+1}=\sum_{k=2}^{n+1} \frac{(-1)^{k}}{k} \sum_{i_{1}+\cdots+i_{k}=n+1} x_{i_{1}} x_{i_{2}} \cdots x_{i_{k}} .
$$

For $k=1,2, \cdots, n$, we set $v_{k}=u_{k}^{k}$. Then (5) can be rewritten as

$$
\begin{aligned}
& x_{k}=\sum_{i_{1}+2 i_{2}+\cdots+k i_{k}=k} \frac{1}{i_{1} ! i_{2} ! \cdots i_{k} !} u_{1}^{i_{1}} u_{2}^{2 i_{2}} \cdots u_{k}^{k i_{k}}, \quad k=1,2, \cdots, n, \\
& x_{n+1}=\sum_{i_{1}+2 i_{2}+\cdots+n i_{n}=n+1} \frac{1}{i_{1} ! i_{2} ! \cdots i_{n} !} u_{1}^{i_{1}} u_{2}^{2 i_{2}} \cdots u_{n}^{n i_{n}} .
\end{aligned}
$$

Using Taylor expansion we obtain

$$
\begin{aligned}
e^{u_{1}+u_{2}^{2}+\cdots+u_{n}^{n}}= & e^{u_{1}} e^{u_{2}^{2}} \cdots e^{u_{n}^{n}} \\
= & \left(1+u_{1}+\frac{u_{1}^{2}}{2 !}+\cdots\right)\left(1+u_{2}^{2}+\frac{u_{2}^{2 \times 2}}{2 !}+\cdots\right) \cdots\left(1+u_{n}^{n}+\frac{u_{n}^{2 \times n}}{2 !}+\cdots\right) \\
= & 1+u_{1}+\left(\frac{u_{1}^{2}}{2 !}+u_{2}^{2}\right)+\left(\frac{u_{1}^{3}}{3 !}+u_{1} u_{2}^{2}+u_{3}^{3}\right)+\cdots \\
= & 1+\sum_{k=1}^{n} \sum_{i_{1}+2 i_{2}+\cdots+k i_{k}=k} \frac{1}{i_{1} ! i_{2} ! \cdots i_{k} !} u_{1}^{i_{1}} u_{2}^{2 i_{2}} \cdots u_{k}^{k i_{k}} \\
& +\sum_{k=n+1}^{\infty} \sum_{i_{1}+2 i_{2}+\cdots+n i_{n}=k} \frac{1}{i_{1} ! i_{2} ! \cdots i_{n} !} u_{1}^{i_{1}} u_{2}^{2 i_{2}} \cdots u_{n}^{n i_{n}} \\
:= & 1+\sum_{k=1}^{\infty} f_{k},
\end{aligned}
$$

where $f_{k}$ is a homogeneous polynomial of degree $k$. Obviously, for $j=1, \cdots, n+1$, we have $f_{j}=x_{j}$, and thus relation (6) is equivalent to

$$
f_{n+1}=\sum_{k=2}^{n+1} \frac{(-1)^{k}}{k} \sum_{i_{1}+\cdots+i_{k}=n+1} f_{i_{1}} f_{i_{2}} \cdots f_{i_{k}} .
$$

On the other hand, from (7) and the fact that $\sum_{k=1}^{\infty} f_{k}$ is positive and small, we get

$$
\begin{aligned}
\sum_{k=1}^{n} u_{k}^{k} & =\ln \left(1+\sum_{k=1}^{\infty} f_{k}\right) \\
& =\left(f_{1}+f_{2}+\cdots\right)-\frac{\left(f_{1}+f_{2}+\cdots\right)^{2}}{2}+\cdots+(-1)^{k-1} \frac{\left(f_{1}+f_{2}+\cdots\right)^{k}}{k}+\cdots .
\end{aligned}
$$


From this and by comparing terms of degree $n+1$ in $\left\{u_{i}\right\}_{1}^{n}$ on both sides, we obtain

$$
\begin{aligned}
0 & =f_{n+1}-\frac{1}{2} \sum_{i_{1}+i_{2}=n+1} f_{i_{1}} f_{i_{2}}+\cdots+\frac{(-1)^{n}}{n+1} \sum_{i_{1}+\cdots+i_{n+1}=n+1} f_{i_{1}} f_{i_{2}} \cdots f_{i_{n+1}} \\
& =f_{n+1}-\sum_{k=2}^{n+1} \frac{(-1)^{k}}{k} \sum_{i_{1}+\cdots+i_{k}=n+1} f_{i_{1}} f_{i_{2}} \cdots f_{i_{k}}
\end{aligned}
$$

and the conclusion follows.

Proof of Proposition 2.1. Lemma 2.1 shows that around $p \in \mathbb{R}^{n+1}$, the Cayley hypersurface can be seen as

$$
F: \mathbb{R}^{n} \supset U \longrightarrow \mathbb{R}^{n+1},
$$

where

$$
\begin{gathered}
F\left(v_{1}, \cdots, v_{n}\right)=\left(v_{1}, v_{2}+\frac{1}{2 !} v_{1}^{2}, \cdots, \sum_{i_{1}+2 i_{2}+\cdots+n i_{n}=n} \frac{1}{i_{1} ! i_{2} ! \cdots i_{n} !} v_{1}^{i_{1}} v_{2}^{i_{2}} \cdots v_{n}^{i_{n}},\right. \\
\left.\sum_{i_{1}+2 i_{2}+\cdots+n i_{n}=n+1} \frac{1}{i_{1} ! i_{2} ! \cdots i_{n} !} v_{1}^{i_{1}} v_{2}^{i_{2}} \cdots v_{n}^{i_{n}}\right) .
\end{gathered}
$$

From (5), direct computations give that

$$
\frac{\partial x_{k}}{\partial v_{j}}=\left\{\begin{array}{ll}
x_{k-j}, & j<k, \\
1, & j=k, \\
0, & j>k,
\end{array} \quad \frac{\partial x_{n+1}}{\partial v_{j}}=x_{n-j+1}, \quad k, j=1, \cdots, n .\right.
$$

Denote by $\frac{\partial}{\partial v_{1}}, \cdots, \frac{\partial}{\partial v_{n}}$ the coordinate vector fields and $F_{*}\left(\frac{\partial}{\partial v_{j}}\right)=F_{v_{j}}$. Then

$$
F_{v_{j}}=(\overbrace{0, \cdots, 0}^{j-1}, 1, x_{1}, \cdots, x_{n-j+1}), \quad j=1, \cdots, n .
$$

Let us take $\xi=(0, \cdots, 0,1)$ as a tentative transversal vector field. For the corresponding volume element $\theta$ we have $\theta\left(\frac{\partial}{\partial v_{1}}, \cdots, \frac{\partial}{\partial v_{n}}\right)=\operatorname{det}\left[F_{v_{1}}, \cdots, F_{v_{n}}, \xi\right]=1$, so that $\left\{\frac{\partial}{\partial v_{1}}, \cdots, \frac{\partial}{\partial v_{n}}\right\}$ is a unimodular basis. From (8) and (9), we have

$$
D_{\frac{\partial}{\partial v_{i}}} F_{v_{j}}= \begin{cases}F_{v_{i+j}}, & i+j<n+1 \\ \xi, & i+j=n+1 \\ 0, & i+j>n+1\end{cases}
$$

Hence, we obtain that

$$
\nabla_{\frac{\partial}{\partial v_{i}}} \frac{\partial}{\partial v_{j}}= \begin{cases}\frac{\partial}{\partial v_{i+j}}, & i+j \leq n, \quad h_{i j}=h\left(\frac{\partial}{\partial v_{i}}, \frac{\partial}{\partial v_{j}}\right)=\left\{\begin{array}{ll}
1, & i+j=n+1 \\
0, & \text { otherwise }
\end{array}\right. \text { otherwise }\end{cases}
$$

Therefore $\operatorname{det}_{\theta} h=\operatorname{det}\left[h_{i j}\right]=(-1)^{n(n-1) / 2}$, so $\xi$ is exactly the affine normal and the Cayley hypersurface is an improper affine hypersphere. From (10) and $C=\nabla h$ we have

$$
C\left(\frac{\partial}{\partial v_{i}}, \frac{\partial}{\partial v_{j}}, \frac{\partial}{\partial v_{l}}\right)= \begin{cases}-2, & i+j+l=n+1 \\ 0, & \text { otherwise }\end{cases}
$$


According to $C\left(\frac{\partial}{\partial v_{i}}, \frac{\partial}{\partial v_{j}}, \frac{\partial}{\partial v_{l}}\right)=-2 h\left(K_{\frac{\partial}{\partial v_{i}}} \frac{\partial}{\partial v_{j}}, \frac{\partial}{\partial v_{l}}\right)$ and $\left(h^{i j}\right)=\left(h_{i j}\right)$, we easily get

$$
K_{\frac{\partial}{\partial v_{i}}} \frac{\partial}{\partial v_{j}}=\left\{\begin{array}{lc}
\frac{\partial}{\partial v_{i+j}}, & i+j \leq n \\
0, & \text { otherwise. }
\end{array}\right.
$$

From $\hat{\nabla}_{X} Y=\nabla_{X} Y-K_{X} Y$ we then obtain $\hat{\nabla}_{\frac{\partial}{\partial v_{i}}} \frac{\partial}{\partial v_{j}}=0$ for all $i, j$.

It follows that the Cayley hypersurface is a flat improper affine hypersphere and satisfies $\hat{\nabla} C=0$. From (11), we get $\left(K_{\frac{\partial}{\partial v_{1}}}\right)^{n-1} \frac{\partial}{\partial v_{1}}=\frac{\partial}{\partial v_{n}}$. Hence $K^{n-1} \neq 0$.

\section{A Differential GeOMEtric CharaCterization OF CAYLEY HYPERSURFACES}

In this section we want to show the converse, thus completing the proof of the Main Theorem.

Proposition 3.1. Let $M$ be a non-degenerate affine hypersurface of $\mathbb{R}^{n+1}$ with constant affine sectional curvature $a$ and $\hat{\nabla} C=0$. Then either $M$ is a quadric (i.e. $C=0)$ or $M$ is a flat affine hypersphere.

Proof. From the assumption, we have

$$
\hat{R}(X, Y) Z=a(h(Y, Z) X-h(X, Z) Y)
$$

whereas the condition $\hat{\nabla} C=0$, or equivalently $\hat{\nabla} K=0$, implies that $M$ is an affine hypersphere [1] and that for all $X, Y, Z$ and $W$, we have

$$
\hat{R}(X, Y) K(Z, W)=K(\hat{R}(X, Y) Z, W)+K(Z, \hat{R}(X, Y) W) .
$$

Let us choose a local orthonormal basis $\left\{e_{1}, \cdots, e_{n}\right\}$ such that $h\left(e_{i}, e_{j}\right)=\epsilon_{i} \delta_{i j}$ and $\epsilon_{i}= \pm 1$. Then from (13), we get

$$
h\left(e_{i}, \hat{R}\left(e_{i}, e_{j}\right) K\left(e_{i}, e_{i}\right)\right)=2 h\left(e_{i}, K\left(\hat{R}\left(e_{i}, e_{j}\right) e_{i}, e_{i}\right)\right),
$$

and (12) then implies that for $i \neq j$,

$$
0=h\left(e_{i}, \hat{R}\left(e_{i}, e_{j}\right) K_{e_{i}} e_{i}-2 K_{e_{i}} \hat{R}\left(e_{i}, e_{j}\right) e_{i}\right)=3 a \epsilon_{i} h\left(K_{e_{i}} e_{i}, e_{j}\right) .
$$

This shows that we have either $a=0$ or $a \neq 0$ and $K_{e_{i}} e_{i}=\mu_{i} e_{i}$ for all $i$. In the latter case, the apolarity condition implies that $\mu_{j}=\operatorname{tr} K_{e_{j}}=0$ for each $j$. Therefore, we get $K=0$ and thus $C=0$.

Let us now consider a non-degenerate affine hypersurface $M$ of $\mathbb{R}^{n+1}$ which has constant affine sectional curvature and satisfies the conditions $\hat{\nabla} C=0, J=0$ and $K^{n-1} \neq 0$. From (3), (4) and Proposition 3.1, we see that $M$ is a flat improper affine hypersphere which satisfies $K_{X} K_{Y}=K_{Y} K_{X}$ for all $X$ and $Y$. We will need the following.

Lemma 3.1 (Lemma 3.3 of [5]). If $K_{X} K_{Y}=K_{Y} K_{X}$, then $K_{X}$ is nilpotent. In particular, $\left(K_{X}\right)^{n}=0$.

Having that $K \neq 0$ and $K_{X} K_{Y}=K_{Y} K_{X}$ for all $X$ and $Y$, we can define, for every natural number $m$, a totally symmetric tensor $K^{m}$ by

$$
K^{m}\left(X_{1}, \cdots, X_{m}, X_{m+1}\right)=K_{X_{1}} K_{X_{2}} \cdots K_{X_{m}} X_{m+1} .
$$


Then $K^{m}$ vanishes identically if and only if $\left(K_{v}\right)^{m} v=0$ for any vector $v$. By assumption, $K^{n-1} \neq 0$ at a point $p$. Then (see Remark 3.3 in [5]), for any tangent vector $v$ at $p$, we have $\left(K_{v}\right)^{n} v=0$ and there exists a tangent vector $u$ at $p$ such that $h\left(\left(K_{u}\right)^{n-1} u, u\right) \neq 0$.

Define

$$
y_{1}=u, \quad y_{i}=\left(K_{u}\right)^{i-1} u, \quad i=2, \cdots, n .
$$

Then $y_{n} \neq 0$. The facts $K_{u} y_{i}=y_{i+1}$ and $K_{u} y_{n}=0$ imply that $y_{1}, y_{2}, \cdots, y_{n}$ are $n$ linearly independent vectors. Since $K_{u}$ is a symmetric operator with respect to $h$, we also get that

$$
h\left(y_{i}, y_{j}\right)= \begin{cases}h\left(y_{i+j-1}, y_{1}\right), & i+j \leq n+1, \\ 0, & \text { otherwise. }\end{cases}
$$

Put $\alpha_{k}=h\left(y_{k}, y_{1}\right), 1 \leq k \leq n$. As $\alpha_{n}=h\left(\left(K_{u}\right)^{n-1} u, u\right) \neq 0$, by re-scaling $u$, and if necessary changing the sign of $\xi$, we may assume that $\alpha_{n}=1$.

Lemma 3.2. Under conditions as stated in the Main Theorem, $M$ is a flat improper affine hypersphere. Moreover, if $K^{n-1} \neq 0$ at $p \in M$, then there exists a basis $\left\{x_{1}, \cdots, x_{n}\right\}$ of $T_{p} M$ such that

$$
K_{x_{i}} x_{j}=\left\{\begin{array}{ll}
x_{i+j}, & i+j \leq n, \\
0, & \text { otherwise }
\end{array} \quad h\left(x_{i}, x_{j}\right)= \begin{cases}1, & i+j=n+1 \\
0, & \text { otherwise }\end{cases}\right.
$$

Proof. The first assertion is a consequence of Proposition 3.1. To prove the second assertion, we choose $u \in T_{p} M$ such that $h\left(\left(K_{u}\right)^{n-1} u, u\right)=1$. Let $y_{1}, \cdots, y_{n}$ be the basis of $T_{p} M$ as constructed in (15). Given an arbitrary real number $\beta$, set $x_{1}=y_{1}+\beta y_{2}$ and $x_{j}=K_{x_{1}} x_{j-1}$ for all $j=2, \cdots, n$. Then

$$
\begin{aligned}
& x_{2}=y_{2}+2 \beta y_{3}+\cdots, x_{3}=y_{3}+3 \beta y_{4}+\cdots, \cdots, \\
& x_{n-1}=y_{n-1}+(n-1) \beta y_{n}, x_{n}=y_{n} .
\end{aligned}
$$

It follows that $h\left(x_{1}, x_{n}\right)=h\left(y_{1}, y_{n}\right)=1$ and $h\left(x_{1}, x_{n-1}\right)=h\left(y_{1}, y_{n-1}\right)+n \beta$. Then we can choose $\beta$ such that $h\left(x_{1}, x_{n-1}\right)=0$. Hence, we may assume that $\alpha_{n-1}=0$.

Next we assume that we have a basis $\left\{y_{1}, \cdots, y_{n}\right\}$ for which $\alpha_{n}=1$ and $\alpha_{n-1}=$ $\cdots=\alpha_{n-i}=0$, for some $i \geq 1$. Then by putting $x_{1}=y_{1}+\beta y_{2+i}$ and $x_{j}=K_{x_{1}} x_{j-1}$ for all $j=2, \cdots, n$, we get

$$
\begin{aligned}
& x_{2}=y_{2}+2 \beta y_{3+i}+\cdots, \\
& x_{3}=y_{3}+3 \beta y_{4+i}+\cdots, \cdots, \\
& x_{n-j}=y_{n-j}+(n-j) \beta y_{n-j+i+1}+\cdots, \cdots, \\
& x_{n-i-1}=y_{n-i-1}+(n-i-1) \beta y_{n}, \\
& x_{n-i}=y_{n-i}, \cdots, x_{n}=y_{n} .
\end{aligned}
$$

It is easily seen that

$$
\begin{aligned}
& h\left(x_{1}, x_{n}\right)=h\left(y_{1}, y_{n}\right)=\alpha_{n}=1, \\
& h\left(x_{1}, x_{n-j}\right)=h\left(y_{1}, y_{n-j}\right)=\alpha_{n-j}=0,1 \leq j \leq i, \\
& h\left(x_{1}, x_{n-i-1}\right)=h\left(y_{1}, y_{n-i-1}\right)+(n-i) \beta .
\end{aligned}
$$

Hence, by choosing $\beta$ appropriately, we may also assume that $\alpha_{n-i-1}=0$.

Continuing this process, using $K_{y_{i}} y_{j}=\left(K_{y_{1}}\right)^{i+j-1} y_{1}$ and (16), we complete the proof. 
Remark 3.1. The second assertion of Lemma 3.2 is similar to Dillen and Vrancken's Lemma 6.1 in [5], where the conclusion is obtained under the conditions $\nabla K=0$ and $K^{n-1} \neq 0$.

Proof of the Main Theorem. Lemma 3.2 shows that $M$ is an $h$-flat improper affine hypersphere. As $M$ is flat, we can extend the basis chosen in Lemma 3.2 to local parallel flat coordinate vector fields $\frac{\partial}{\partial v_{1}}, \cdots, \frac{\partial}{\partial v_{n}}$, with corresponding coordinates $\left(v_{1}, \cdots, v_{n}\right)$, the point $p$ corresponding to 0 . Then we have

$$
h\left(\frac{\partial}{\partial v_{i}}, \frac{\partial}{\partial v_{j}}\right)= \begin{cases}1, & i+j=n+1, \\ 0, & \text { otherwise. }\end{cases}
$$

Set

$$
K\left(\frac{\partial}{\partial v_{i}}, \frac{\partial}{\partial v_{j}}\right)=\sum_{\ell=1}^{n} A_{i j}^{\ell} \frac{\partial}{\partial v_{\ell}}
$$

Since $\hat{\nabla} K=0$ and $\hat{\nabla} \frac{\partial}{\partial v_{i}} \frac{\partial}{\partial v_{j}}=0$, the initial conditions (17) at $p$ shows that $A_{i j}^{\ell}$ are all constant and satisfy

$$
A_{i j}^{\ell}= \begin{cases}1, & \ell=i+j \leq n \\ 0, & \text { otherwise }\end{cases}
$$

Denote $\frac{\partial^{k} F}{\partial v_{i_{1}} \cdots \partial v_{i_{k}}}=F_{v_{i_{1}} \cdots v_{i_{k}}}$. Due to the fact that the affine normal $\xi$ of improper affine hypersphere is constant and that $D_{X} Y=K_{X} Y+\hat{\nabla}_{X} Y+h(X, Y) \xi$, the immersion $F$ is determined by the following system of differential equations:

$$
F_{v_{i} v_{j}}= \begin{cases}F_{v_{i+j}}, & i+j<n+1 \\ \xi, & i+j=n+1 \\ 0, & i+j>n+1\end{cases}
$$

This is equivalent to

$$
F_{v_{i_{1}} \cdots v_{i_{k}}}= \begin{cases}F_{{v_{1}+\cdots+i_{k}}_{1},}, & i_{1}+\cdots+i_{k}<n+1 \\ \xi, & i_{1}+\cdots+i_{k}=n+1 \\ 0, & i_{1}+\cdots+i_{k}>n+1\end{cases}
$$

Set $A_{n+1}=\xi$ and $A_{i}=F_{v_{i}}(0)=y_{i}$ for all $i=1, \cdots, n$. Then

$$
F_{v_{i_{1}} \cdots v_{i_{k}}}(0)=A_{i_{1}+\cdots+i_{k}}, \quad \text { if } i_{1}+\cdots+i_{k} \leq n+1 .
$$

By using Taylor expansion and (18), we obtain, up to an affine translation,

$$
\begin{aligned}
F\left(v_{1}, \cdots, v_{n}\right)= & \left.\left(\sum_{i=1}^{n} v_{i} \frac{\partial}{\partial v_{i}}\right) F\right|_{0}+\left.\frac{1}{2 !}\left(\sum_{i=1}^{n} v_{i} \frac{\partial}{\partial v_{i}}\right)^{2} F\right|_{0}+\cdots \\
& +\left.\frac{1}{n !}\left(\sum_{i=1}^{n} v_{i} \frac{\partial}{\partial v_{i}}\right)^{n} F\right|_{0}+\left.\frac{1}{(n+1) !}\left(\sum_{i=1}^{n} v_{i} \frac{\partial}{\partial v_{i}}\right)^{n+1} F\right|_{0} .
\end{aligned}
$$


By using (18), we easily see the following:

$$
\begin{aligned}
\left.\left(\sum_{i=1}^{n} v_{i} \frac{\partial}{\partial v_{i}}\right) F\right|_{0} & =\sum_{i=1}^{n} v_{i} A_{i}, \\
\left.\frac{1}{\ell !}\left(\sum_{i=1}^{n} v_{i} \frac{\partial}{\partial v_{i}}\right)^{\ell} F\right|_{0} & =\frac{1}{\ell !} \sum_{k=\ell}^{n+1}\left(\sum_{j_{1}+j_{2}+\cdots+j_{\ell}=k} v_{j_{1}} v_{j_{2}} \cdots v_{j_{\ell}}\right) A_{k} \\
& =\sum_{k=\ell}^{n+1}\left(\sum_{\substack{i_{1}+\cdots+i_{n}=\ell \\
i_{1}+2 i_{2}+\cdots+n i_{n}=k}} \frac{1}{i_{1} ! i_{2} ! \cdots i_{n} !} v_{1}^{i_{1}} v_{2}^{i_{2}} \cdots v_{n}^{i_{n}}\right) A_{k}, \quad 2 \leq \ell \leq n, \\
\left.\left(\sum_{i=1}^{n} v_{i} \frac{\partial}{\partial v_{i}}\right)^{n+1} F\right|_{0} & =v_{1}^{n+1} A_{n+1} .
\end{aligned}
$$

From the above calculations, the right hand side of (19) becomes a combination of $A_{n+1}$ and $A_{k}(k=1, \cdots, n)$ with coefficients as follows:

$$
\sum_{\ell=2}^{n+1} \sum_{\substack{i_{1}+\cdots+i_{n}=\ell \\ i_{1}+2 i_{2}+\cdots+n i_{n}=n+1}} \frac{1}{i_{1} ! i_{2} ! \cdots i_{n} !} v_{1}^{i_{1}} v_{2}^{i_{2}} \cdots v_{n}^{i_{n}}=\sum_{i_{1}+2 i_{2}+\cdots+n i_{n}=n+1} \frac{1}{i_{1} ! i_{2} ! \cdots i_{n} !} v_{1}^{i_{1}} v_{2}^{i_{2}} \cdots v_{n}^{i_{n}}
$$

and

$$
\begin{aligned}
\sum_{\ell=1}^{k} \sum_{\substack{i_{1}+\cdots+i_{n}=\ell \\
i_{1}+2 i_{2}+\cdots+n i_{n}=k}} \frac{1}{i_{1} ! i_{2} ! \cdots i_{n} !} v_{1}^{i_{1}} v_{2}^{i_{2}} \cdots v_{n}^{i_{n}} & =\sum_{\ell=1}^{k} \sum_{\substack{i_{1}+\cdots+i_{k}=\ell \\
i_{1}+2 i_{2}+\cdots+k i_{k}=k}} \frac{1}{i_{1} ! i_{2} ! \cdots i_{k} !} v_{1}^{i_{1}} v_{2}^{i_{2}} \cdots v_{k}^{i_{k}} \\
& =\sum_{i_{1}+2 i_{2}+\cdots+k i_{k}=k} \frac{1}{i_{1} ! i_{2} ! \cdots i_{k} !} v_{1}^{i_{1}} v_{2}^{i_{2}} \cdots v_{k}^{i_{k}}
\end{aligned}
$$

where we use the fact that all indices $i_{1}, \cdots, i_{n}$ are non-negative integers. Hence, we can finally rewrite (19) as follows:

$$
\begin{aligned}
F\left(v_{1}, \cdots, v_{n}\right)= & v_{1} A_{1}+\left(v_{2}+\frac{1}{2 !} v_{1}^{2}\right) A_{2}+\left(v_{3}+v_{2} v_{1}+\frac{1}{3 !} v_{1}^{3}\right) A_{3}+\cdots \\
& +\left(\sum_{i_{1}+2 i_{2}+\cdots+k i_{k}=k} \frac{1}{i_{1} ! i_{2} ! \cdots i_{k} !} v_{1}^{i_{1}} v_{2}^{i_{2}} \cdots v_{k}^{i_{k}}\right) A_{k}+\cdots \\
& +\left(\sum_{i_{1}+2 i_{2}+\cdots+n i_{n}=n} \frac{1}{i_{1} ! i_{2} ! \cdots i_{n} !} v_{1}^{i_{1}} v_{2}^{i_{2}} \cdots v_{n}^{i_{n}}\right) A_{n} \\
& +\left(\sum_{i_{1}+2 i_{2}+\cdots+n i_{n}=n+1} \frac{1}{i_{1} ! i_{2} ! \cdots i_{n} !} v_{1}^{i_{1}} v_{2}^{i_{2}} \cdots v_{n}^{i_{n}}\right) A_{n+1} .
\end{aligned}
$$

Since $A_{1}, \cdots, A_{n+1}$ are constant linearly independent vectors, up to an affine transformation, we can write

$$
\begin{aligned}
& F\left(v_{1}, \cdots, v_{n}\right)=\left(v_{1}, v_{2}+\frac{1}{2 !} v_{1}^{2}, \cdots, \sum_{i_{1}+2 i_{2}+\cdots+n i_{n}=n} \frac{1}{i_{1} ! i_{2} ! \cdots i_{n} !} v_{1}^{i_{1}} v_{2}^{i_{2}} \cdots v_{n}^{i_{n}},\right. \\
& \left.\sum_{i_{1}+2 i_{2}+\cdots+n i_{n}=n+1} \frac{1}{i_{1} ! i_{2} ! \cdots i_{n} !} v_{1}^{i_{1}} v_{2}^{i_{2}} \cdots v_{n}^{i_{n}}\right) .
\end{aligned}
$$

According to Lemma 2.1, $M$ is locally affine equivalent to the Cayley hypersurface. 


\section{REFERENCES}

[1] N. Bokan, K. Nomizu and U. Simon, Affine hypersurfaces with parallel cubic forms, Tôhoku Math. J. 42 (1990), 101-108. MR1036477 (90m:53018)

[2] Y. Choi and H. Kim, A characterization of Cayley hypersurface and Eastwood and Ezhov conjecture, Internat. J. Math. 16 (2005), 841-862. MR/2168070 (2006g:53075)

[3] F. Dillen and L. Vrancken, 3-dimensional affine hypersurfaces in $\mathbb{R}^{4}$ with parallel cubic form, Nagoya Math. J. 124 (1991), 41-53. MR1142975 (92m:53016)

[4] F. Dillen and L. Vrancken, Generalized Cayley surfaces, Proceedings of the Conference on Global Analysis and Global Differential Geometry, Berlin, 1990, Lecture Notes in Mathematics, 1481, Springer, 1991, 36-47. MR1178516 (93m:53011)

[5] F. Dillen and L. Vrancken, Hypersurfaces with parallel difference tensor, Japan J. Math. (N.S.) 24 (1998), 43-60. MR.1630113 (99e:53013)

[6] F. Dillen, L. Vrancken and S. Yaprak, Affine hypersurfaces with parallel cubic form, Nagoya Math. J. 135 (1994), 153-164. MR1295822 (95f:53096)

[7] M. Eastwood and V. Ezhov, Cayley hypersurfaces, Proc. Steklov Inst. Math. 253 (2006), 221-224. MR2338700 (2008i:53012)

[8] Z.J. Hu and C.C. Li, The classification of 3-dimensional Lorentzian affine hypersurfaces with parallel cubic form, preprint (2010).

[9] Z.J. Hu, H. Li, U. Simon and L. Vrancken, On locally strongly convex affine hypersurfaces with parallel cubic form. I, Diff. Geom. Appl. 27 (2009), 188-205. MR2503972|(2010b:53015)

[10] Z.J. Hu, H. Li and L. Vrancken, Characterizations of the Calabi product of hyperbolic affine hyperspheres, Results in Math. 52 (2008), 299-314. MR2443493 (2009i:53006)

[11] Z.J. Hu, H. Li and L. Vrancken, Locally strongly convex affine hypersurfaces with parallel cubic form, J. Diff. Geom., to appear.

[12] M. Magid and K. Nomizu, On affine surfaces whose cubic forms are parallel relative to the affine metric, Proc. Japan Acad. Ser. A Math. Sci. 65 (1989), 215-218. MR.1030183 (90m:53021)

[13] K. Nomizu and U. Pinkall, Cayley surfaces in affine differential geometry, Tôhoku Math. J. (2) 41 (1989), 589-596. MR1025324 (90j:53010)

[14] K. Nomizu and T. Sasaki, Affine differential geometry, Cambridge University Press, 1994. MR1311248 (96e:53014)

[15] L. Vrancken, Affine higher order parallel hypersurfaces, Ann. Fac. Sci. Toulouse Math. (5) 9 (1988), 341-353. MR1425270 (97g:53073)

[16] L. Vrancken, The Magid-Ryan conjecture for equiaffine hyperspheres with constant sectional curvature, J. Diff. Geom. 54 (2000), 99-138. MR1815413 (2001m:53022)

Department of Mathematics, Zhengzhou University, Zhengzhou 450052, People's RePublic of China

E-mail address: huzj@zzu.edu.cn

Department of Mathematics, Zhengzhou University, Zhengzhou 450052, People's REPUBLIC OF CHINA

E-mail address: ceceli@sina.com

Department of Mathematics, Zhengzhou University, Zhengzhou 450052, People's Republic of China

E-mail address: zd20082100333@163.com 